\title{
Geochemical barriers as a form of self- organization of geochemical systems (case of study Kerch Peninsula, Russia)
}

\author{
Aleksandr Khaustov $^{1, *}$, Margarita Redina $^{1}$, and Eugeniya Yakovleva $^{2}$ \\ ${ }^{1}$ Peoples' Friendship University of Russia, Russia \\ ${ }^{2}$ Institute of Biology, Komi Research Centre of RAS, Russia
}

\begin{abstract}
According to the author's concept, discharge of groundwater to the surface is considered as a sharp change of geochemical conditions, leading to the formation of geochemical barriers (GCB). In the article presented results of geochemical study of groundwater-soil-organic mattergas interaction as example of Kerch Peninsula self-organizing system The geochemical barriers give rise to the formation of a kind of nonequilibrium geoecosystems with an individual structure of direct and inverse connections between the components, size and borders of geochemical systems (GCS) are determined by many factors: frequency and scale of discharge, chemical composition, temperature of the source and of the environment, phase transitions within the system with interacting heterogeneous media: "water - rocks", "water - atmosphere", "water - ice", "water - soil", "water - plants (including algae and other living organisms)", and etc. Polycyclic aromatic hydrocarbons (PAHs) are used as tracers of this interaction.
\end{abstract}

\section{Introduction}

Groundwater discharge to the earth's surface can be considered as energy supplying and converting components of systems with non-equilibrium structure fluctuations. We do not exclude the flow of energy and substances from the environment into the components of the aquatic system, as well as the resulting heat and secondary mass flow (i.e., due to the reactions of oxidation of $\mathrm{H}_{2} \mathrm{~S}$ to water and sulfur) [1]. In this formulation, groundwater sources and mud volcanoes are considered as a "biotechnological reactor" for the evolution of equilibrium aquatic ecosystems. The nonequilibrium leads to the emergence of coherent energy and/ or substance flows. The ordering of the state of the system develops against the background of disequilibrium. There is a kind of confrontation between the processes of transfer of matter and energy, breaking the equilibrium, and the internal (relaxation) processes, seeking to restore it. The concept of "aquatic geosystem" implies a common space where the components of water bodies are in system communication with each other.

The goal of the study is to assess the evolution of the system and the diversity of its components interaction. The solution of this geochemical problem is of particular interest,

* Corresponding author: akhaustov@yandex.ru 
since the behavior of organic substances on geochemical barriers has been studied very poorly.

\section{Object of study}

Taking into account the processes of formation of groundwater in the region of the Kerch peninsula, 4 types of groundwater discharges were investigated.

Point 1. Mud methane volcano Ararat with active and constant mixing of mechanical suspensions without carrying them to the surface. In fluids, content of water is less than of solid material; their genesis is determined by the periodic flow of water and gases from the horizons of the deep foundation of the Azov - Black Sea artesian basin. The origin of methane is associated with a gas hydrate deposit. Sampling at point 1 was carried out at different distances from the volcano vent. The $1^{\text {st }}$ sample of soil and plants was taken directly near the source, the $2^{\text {nd }}$ about a meter further, the $3^{\text {rd }}$ about $3 \mathrm{~m}$ from the volcano.

Point 2. Hydrosulphuric subthermal source in the area of the old mud baths; spreads on the shore of lake Chokrak with a sharp change from the reducing situation to oxidizing. It forms a sulfide geochemical zone in the brine of the lake. Samples were taken within a clearly defined $\mathrm{H}_{2} \mathrm{~S}$ oxidation zone from the discharge to the contact with the lake edge.

Point 3. The unique lake Chokrak is a salt lake detached from the sea, fed by hydrogen sulfide springs and Azov sea waters. A sample of rapa, salt crystals, bottom sediments was collected on a dam of Chokrak lake outside the influence of the source

Point 4. Ascending bland cold spring in Babchik gully with the manifestation of consortia (a set of plant populations inherent in this aquatic biogeocenosis) and aquatic vegetation (miriophyllum); there is a slight smell of $\mathrm{H}_{2} \mathrm{~S}$. Samples were taken directly at the exit and at a distance of no more than $5 \mathrm{~m}$.

\section{Methods}

Because of the variety of organic indicator substances, we analyzed the variation of PAH concentrations for discharges with different types of water in the system of their interaction with silty sediments and soils, plant communities in the zone of influence of water and plant consortia. As tracers (indicators of processes of the formation of barriers), the concentrations of polycyclic aromatic hydrocarbons (PAHs) in the components of geochemical systems are used: Naphthalene (Naph), Acenaphthene (Ace), Fluorene (Fluorene), Phenanthrene (Phen), Anthracene (An), Fluoranthene (Flu), Pyrene (Py), Chrysene (Chr), Benzo[a]anthracene (BaA), Benzo[a]pyrene (BaP), Benzo[b]fluoranthene $(\mathrm{BbFlu})$, Perylene (Pl), Benzo[k]fluoranthene (BkFlu), Benzo[ghi]perylene (Bghi), Indeno[1,2,3-cd]pyrene (IP), Dibenzo[a,h]anthracene (DBa). Thos is due to their indicative properties, relative stability and high toxicity. And, the most important, certain combinations of PAHs in the natural and technogenic objects indicates probable source of the pollution. Generally is accepted the following division of PAHs according to their genesis: $\mathrm{Py}$ and $\mathrm{BaP}$ are typical for objects of pyrogenic nature; for naftigenesis - accumulation and transformation of Naph, Phen; for biogenesis, Phen, Chr, and Pl [2]. Due to their structural features, PAHs reflect most representatively the essence of the kinetics of the processes of vertical and horizontal migration of both natural and man-made flows of substances.

To assess the individuality of migration, the concentration ratios (CC) of PAHs were used as a ratio of the PAH concentration in the receiving component (a medium contacting with a water) and to its concentration in water. $\mathrm{CC} \geq 1$ corresponds to the accumulation of matter.

To assess the intensity of the geochemical processes calculations of the increase of entropy of a PAHs set on the barrier were used: the higher is the $\Delta \mathrm{S}$ value for the contacting media, the 
more intensive is a process.

\section{Results and Discussion}

Table 1 shows the initial composition of PAHs in water of studied objects. The composition of dissolved PAHs is interesting because they are extremely rarely determined in groundwater.

Table 1. PAHs content in the water samples, $\mathrm{ng} / \mathrm{dm}^{3}$.

\begin{tabular}{|c|c|c|c|c|}
\hline Point & $\mathbf{1}$ & $\mathbf{2}$ & $\mathbf{3}$ & $\mathbf{4}$ \\
\hline Naph & 82.0 & 51.7 & 7985.2 & 93.2 \\
\hline Ace & 4.5 & 5.2 & 328.4 & 0 \\
\hline Fluorene & 2.7 & 1.5 & 214.2 & 0 \\
\hline Phen & 0 & 6.6 & 429.3 & 3.5 \\
\hline Flu & 3.3 & 1.4 & 42.8 & 0.9 \\
\hline Py & 3.4 & 2.1 & 59.0 & 0.9 \\
\hline An & 0 & 0 & 389.1 & 0 \\
\hline BaA & 0 & 0 & 0 & 0.4 \\
\hline Chr & 0 & 0 & 12.5 & 0.6 \\
\hline BaP & 1.5 & 0.3 & 0 & 0 \\
\hline ¿PAHs & 97.4 & 68.8 & 9460.5 & 99.5 \\
\hline
\end{tabular}

The individual structure of the interaction between components of aquatic systems is clearly visible on the basis of migration flows of PAHs. When discharging on the surface, groundwater contact first with silt (pelitic fraction with pronounced sorption properties). The specificity of chemical types of groundwater has clearly affected the chains of intensity of interaction in the system "water-adjacent media" (Table 2).

Table 2. Activity of transition of PAH in the aquatic components of the system.

\begin{tabular}{|c|c|c|c|c|}
\hline GCB & $\begin{array}{c}\text { Sampling } \\
\text { point }\end{array}$ & $\begin{array}{c}\begin{array}{c}\text { PAH accumulation activity } \\
\text { series }\end{array} \\
\end{array}$ & $\begin{array}{c}\text { Accumulation } \\
\text { limits }\end{array}$ & $\begin{array}{l}\Delta S \text { on the } \\
\text { GCB } \\
\end{array}$ \\
\hline \multirow[t]{3}{*}{ Water - silt } & 1 & $\begin{array}{l}\text { Py }>\text { Flu }>\text { Fluorene }>\text { Ace }>\text { Naph } \\
>\mathrm{BaP}\end{array}$ & $2.0 \cdot 10^{6}$ & -46.5 \\
\hline & 2 & $\mathrm{BaP}>\mathrm{Flu}>\mathrm{Py}>\mathrm{Phen}>\mathrm{Naph}$ & $7.5 \cdot 10^{6}$ & -35.6 \\
\hline & 4 & Flu $>$ Py $>$ Chry $>$ Phen $>$ Naph & $27.9 \cdot 10^{6}$ & -5.1 \\
\hline \multirow[t]{2}{*}{ Water-soil } & 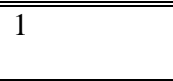 & $\begin{array}{l}\text { Flu >Py > BaP > Fluorene }>\text { Ace } \\
>\text { Naph }\end{array}$ & $3.0 \cdot 10^{6}$ & -69.7 \\
\hline & 2 & $\begin{array}{l}\text { Flu }>\mathrm{BaP}>\text { Py }>\text { Fluorene }>\text { Phen }> \\
\text { Ace }>\text { Naph }\end{array}$ & $4.7 \cdot 10^{6}$ & -50.1 \\
\hline \multirow{2}{*}{$\begin{array}{l}\text { Water- } \\
\text { consortium; } \\
\text { Water - } \\
\text { miriofillyum }\end{array}$} & $\begin{array}{l}\text { 4: Water - } \\
\text { consortium }\end{array}$ & $\begin{array}{l}\text { Py }>\text { Flu }>\text { Phen }>\text { Chr }>\mathrm{BaA}> \\
\text { Naph }\end{array}$ & $19.6 \cdot 10^{6}$ & +123.8 \\
\hline & $\begin{array}{l}\text { 4: Water - } \\
\text { miriofillyum }\end{array}$ & $\begin{array}{l}\text { Flu }>\text { Py }>\text { Phen }>\text { Chr }>\mathrm{BaA}> \\
\text { Naph }\end{array}$ & $35.6 \cdot 10^{6}$ & +115.2 \\
\hline \multirow[t]{2}{*}{$\begin{array}{l}\text { Water - plant } \\
\text { (roots) }\end{array}$} & 1 & $\begin{array}{l}\text { Py }>\text { Flu }>\text { Fluorene }>\text { BaP }>\text { Ace } \\
>\text { Naph }\end{array}$ & $1.9 \cdot 10^{6}$ & -104.6 \\
\hline & 2 & $\begin{array}{l}\text { Flu }>\text { Fluorene }>\text { Phen }>\text { BaP }>\text { Py } \\
>\text { Ace }>\text { Naph }\end{array}$ & $19.0 \cdot 10^{6}$ & +147.6 \\
\hline \multirow{2}{*}{$\begin{array}{l}\text { Soil - plant } \\
\text { (roots) }\end{array}$} & 1 & Ace >Phen & 1.06 & +24.5 \\
\hline & 2 & $\begin{array}{l}\text { Phen }>\mathrm{An}>\mathrm{Naph}=\text { Fluorene }> \\
\text { Flu }>\mathrm{Chr}>\mathrm{Py}>\text { Ace }>\mathrm{BaA}>\mathrm{BaP}\end{array}$ & 7.75 & +197.7 \\
\hline \multirow[t]{2}{*}{$\begin{array}{l}\text { Plant (roots)- } \\
\text { aerial part }\end{array}$} & 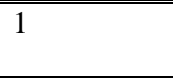 & $\begin{array}{l}\mathrm{Py}>\mathrm{Chr}>\mathrm{BaP}>\mathrm{BaA}>\mathrm{An}> \\
\text { Naph }>\text { Flu }>\text { Phen }\end{array}$ & 2.37 & +59.5 \\
\hline & 2 & Ace $>\mathrm{Py}>(\mathrm{BaA})$ & 1.13 & -126.4 \\
\hline
\end{tabular}


For methane waters of the mud volcano (point 1), as well as for other water bodies, the secondary biogenic genesis of PAH is clearly traced. This composition continues to evolve, but already in conditions a sharp change of on GCB restorative environment on oxidative. Hence the different types of activity of PAH transition from water to environment. The series are similar, but $\mathrm{CC}$ for active PAHs are 1.5-2 times higher. Most actively moving Flu and Py, less intense Naph, Ace and BaA.

When methane and hydrogen sulfide waters interact with the silt fraction, there is a significant difference in the CC series. In our opinion, this is due to the physicochemical properties and conditions of groundwater discharge. During mud volcanism in the crater there is a constant pulsation of water and intensive autowave processes in the system "water-mineral suspensions". Due to the lipophilic properties of PAHs, Py and Flu transitions dominate here, and Phen and Chr did not show a tendency to sorption on a fine basis. Consequently, not all PAHs tend to sorption on solid suspensions due to lipophilicity, as stated in the literature.

At the point 2 (sulfide waters) the "pyrogenic" BaP is in the lead, although it is believed that it is the most resistant to physicochemical transformations of all PAHs. At the same time, Flu, Py and Chr are typical representatives of the biogenic genesis of PAHs, having the leading position on migration abilities in the fresh source at the point 4 .

The negative relatively small values of the entropy increment for the chain "water silt" indicate that this relationship is relatively stationary, while the maximum of nonequilibrium stationarity (the average value of the entropy increment is 46.5 ) is confined to a pulsating mud volcano. High entropy values and, therefore, the highest level of selforganization in comparison with other objects are due to the fluctuations of exhalation flows. Thus, the point 4 , represented by the discharge of groundwater with a more or less stable debit, has a close to zero entropy. This indicates a possible "under-saturation" of silt with PAHs and, consequently, a greater distance from the geochemical equilibrium. For the three points under study, the entropy increment of the nonequilibrium organization of objects forms the following subordination: $\Delta \mathrm{S} 1>\Delta \mathrm{S} 2>\Delta \mathrm{S} 4$.

For some samples in the "water - silt" structure at point 4 the maximum positive entropy values were revealed. This may indicate the maximum saturation of the medium receiving the PAHs (up to $290 \mathrm{ng} / \mathrm{kg}$ ). In the chemical composition dominate: Phen, Py, Fluorene, Chr, Naph, BbFlu, BaP. Such a wide range of PAHs and their sum convincingly confirm again: their maximum concentrations can be due to the specific adsorption of these substances to many surfaces (to fine silts, most saturated with organic matter - even more so).

It is important to highlight the growth of absolute and relative entropy in the aquatic vegetation. Theoretically, with such interactions, there is a significant complication of selforganizing structures, which should be accompanied by antientropic or negative values of its production. But we get positive values in our calculations. This may indicate the release of energy into the surrounding space in open nonlinear systems. F.A. Letnikov (1992) admits that some heat can be consumed in irreversible systems (all the dissipative systems are so) for the irreversible increase of entropy, even in abiogenic systems.

Summarizing the results of studies of self-organization processes S.L. Shvartsev [3] identified three types of dissipative systems in which:

1) structures develop from complex to simple with increasing entropy (degrading);

2) structures, developing with a decrease in the entropy production (self-organizing);

3) structures that develop along the line of complication with obtaining negative entropy (non-entropy), and lead to self-organization.

The last type of complication of abiogenic dissipative structures was identified by S.L. Shvartsev. We emphasize that he studied mainly abiogenic systems "water-rock without "living matter". Its inclusion into the GCS as an active component significantly changes our understanding of dissipative GCS at all levels of the organization. 
Therefore, taking into account our experimental data, it is important to allocate another type of natural dissipative structures that develop with the participation of living matter from simple (aqueous solutions) to complex (i.e., hydrophytes) with the production of entropy.

So, on the example of the consortium and the miriophyllum, these (even with small differences) amounts of PAH are very contrasting with the same composition of the initial solution (point 4). For the water consortium, Naph, Phen, Flu, Py and almost all multi ring angular polyarenes prevail (truly, the diversity determines the complexity of the chemical composition of substances!). In miriophyllum lead Phen, Naph, Flu, and Py in the absence of heavy PAHs with the exception of BaP. This most toxic representative of the PAHs was not detected in water on the point 4 (it is very poorly soluble and is given from the solution), but it could get into miriophyllum and into the consortium by aerogenic way.

In soils contacting with the peloid fraction of silt deposits, there is an intensive accumulation of PAHs, not necessarily from filtered mineral waters. Confirmation of this is an experiment with the addition of tritium-labeled 3,4-benzpyrene [4]. A month after the labeling, PAHs were detected in the extracted composition of humic acids, and each soil type has its own quantitative pool of polyarenes. That is, besides the atmospheric genesis, under the influence of its own microbiota, hydrocarbons, being transformed, are included in the composition of humic and fulvic acids.

Soils are most capable of self-regulation in relation to not only PAHs. This is confirmed by almost identical transition series, despite the contrasting differences in the initial composition of the waters, as well as by the entropy calculations in the "water-soil" system. Its negative values for all samples indicate an active interaction and redistribution of PAHs in the root system with aqueous solutions. Despite the constant infiltration into the soils of a certain pool of PAHs, equilibrium in systems with water is not reached. For a mud volcano (point 1) contacts with the water of surrounding soils occur discretely in time (gas streams of dry methane and other hydrocarbons are not excluded). And for sulphate waters (point 2) with their constant contact, we should expect maximum values of $\mathrm{PAH}$ accumulation rates.

When comparing the actual data, the opposite is true. At point 1, more than twofold exceedances of PAHs of predominantly naphtogenic genesis are recorded in soils. These are Naph and Phen, as well as the "exotic" multi-ring BbFlu and Bghi, with the latter PAHs are not presented in the zone of influence of sulphide waters. The relatively small values of the sum of PAHs and the composition of their pool can be explained both by the genesis and (to a greater extent) by microbial oxidation processes. Low PAHs concentrations in the soils of this zone are explained by self-regulation (self-organization) due to this process on the oxygen barrier. PAH migration plays a definite role in the root system of the saltwater and further along the stems, covering large areas in the oxidation zone.

Binding of PAHs in soil waters with humus and fulvic acids is similar to the process of removing PAHs from the dissolved state, which can lead to a change in the equilibrium established between the suspended and dissolved forms of PAHs. The processes are accompanied by an increase in their solubility in water, a decrease in volatility and bioaccumulation, and an acceleration of photolysis in soil horizons [4]. Such interaction of groundwater with soil solutions and soils leads to the accumulation of Flu, Py, BaP, Fluorene, Ace, Naph. However, this pool of polyarenes is not all available for the plant root system (rhizosphere). Many studies are devoted to this problem in connection with the toxic carcinogenic and mutagenic effects of PAH on biota.

In principle, there are two ways of entering substances into plants: the first is through the root system and further along the stem; the second one is through the ground part of the plant from air after they evaporate from the soil and in the direct contact with a dust. It is believed that hydrophobic hydrocarbons interact passively with the plant root system and are mostly sorbed by a hydrophobic cuticle on the surface of the green mass with 
subsequent digestion. In favor of this argument, the values of $\lg \mathrm{K}_{\mathrm{ow}}=3.0-3.5$ are given, above which PAHs do not penetrate the roots, but are firmly bound on their surface. Values of $\lg$ Kow (characterizes the hydrophobicity and polarity of the compounds) for the considered PAHs vary from 3.7 (Naph) to 7.23 (Bghi).

According to the above arguments, the entry of PAHs into plants should occur predominantly by the aerial route. But our studies and studies by other authors showed the fundamental possibility of adsorption by the root system and further migration not only of low molecular weight PAHs, but also of active movement of heavy polyarenes from the soil into plants. This fact is convincingly confirmed by analyzes of samples of saltwater on the point 2, located in the constant zone of impact of the source, in which the values of the sum of PAHs in the root part significantly exceed those in the aerial part of the plant. The difference between the amounts of polyarene and the movement of solutions along the stem can be more than twofold. So, in the root samples, the sum of PAHs was $240 \mathrm{ng} / \mathrm{g}$, and the stem was $121 \mathrm{ng} / \mathrm{g}$; in sample 3, respectively, 153 and $85 \mathrm{ng} / \mathrm{g}$.

An interesting pattern is the practical invariance of the PAHs pool composition when they move along the stem of saltwort (the effect of self-regulation). The value of entropy in the system "soil-plants" at CC 7,75 is +198 . This indicates the nonequilibrium direction of the PAH flow from soils to the rhizosphere of plants. This flow is uneven in time and space, which can be explained by the valve principle of the set of micro-GCB formed on the surface of the root system. Such barriers are selective to the composition of PAH and for existing geochemical situation have a pronounced subordination (Table 2). A certain, if not dominant, role is played by microflora, which may prevent the selective diffusion of organic substances. Almost all plants actively interact with microorganisms capable of destruction of hydrocarbons, including PAH. The number of such oil-oxidizing bacteria according to the literature data [4] can reach $10^{6}-10^{7}$ and even $8.1 \cdot 10^{8} \mathrm{CFU} / \mathrm{g}$.

Then, when moving along the plant stem, due to their physiology (separation of the flow into capillaries, etc.), the energy potential is minimized; this leads to mass flow ordering (similar to the transition from turbulent to laminar). We receive a decrease in fluctuations in the system (which, naturally, affected the decrease in CC to 1.13) and a sharp drop in entropy (-126); this is a confirmation of the sustainable character of structure formation. According to the literature, the values of the PAHs bioconcentration coefficients from the soil can vary from $10^{-6}$ to 16 , and for individual substances it can increase up to $30-40$ [4].

In the area of point 1 , inverse patterns of migration are observed in the system "water soil - vegetation". Due to the weak contact with the water phase of a mud volcano, the concentration of total PAHs in the soils is significantly lower compared to the point 2 . They make up 67-85 ng / $\mathrm{g}$ with prevalence of Fluorene and Naph in the chemical composition, but most importantly, the maximum concentrations of Bghi for all objects from 8 to $10 \mathrm{ng} /$ $\mathrm{g}$ are found. Such a pool is typical for representatives of PAH naftogenesis. However, they did not switch to the root system, but only BaP. Moreover, it is much less in the roots than in the aboveground part of the saltwort, similarly to the behavior of Py and other representatives of PAHs of pyrogenic genesis. So, the sum of PAHs in the root part is 7$68 \mathrm{ng} / \mathrm{g}$, in the above-ground part of the saline is $15-169 \mathrm{ng} / \mathrm{g}$, which indicates the predominantly aerogenic pathway of the pool. The presence of PAHs in the plants of the Kerch Peninsula in the reserve is most likely associated with their release from the atmosphere with subsequent adsorption on plants. The adsorption of hydrocarbons by the stems and leaves of plants from air and water is of great importance for the self-purification of media. According to [4 and others], the aboveground part of plants is able to sorb and accumulate from the air $10^{3}-10^{6}$ times more $\mathrm{PAH}$ than is contained in the atmosphere. For our example, the appearance in the vegetation of DBa, Bghi, Ip (a pool of multiring PAHs) clearly demonstrates pyrogenic about the atmospheric genesis, and small positive values of 
entropy $(+25$ and +60$)$ in the system "soil - roots - stem" indicate the established process and directivity PAH distributions.

In biota, which is directly and permanently in contact with water (consortium and miriophyllum), light PAHs (Naph, Ace) accumulate in much smaller quantities than the Py and Fluorene. Self-regulating processes are also clearly manifested here, but they are more clearly expressed in comparison with soils. Soils can be considered as a universal abiogenic-biogenic filter on the way of solutions in the rhizosphere of plants. Calculations of entropies for short and long chains of interaction between components indicate fundamental differences between these interactions.

The higher entropy values in the roots and especially above-ground parts of the plant are largely due to the presence of relatively easily soluble PAHs with high concentrations: Naph, Fluorene, Py and Flu. These substances accumulate significantly more actively in the aerial part of plants even in comparison with roots, not to mention water. However, the concentration of the most soluble PAH, Naph, in water was 1.5 times higher than in the aerial part of the plant.

As expected, the highest value of the molar entropy recorded in the hydrophytes, draws the substance and energy for their evolution directly from water. Hydrophytes represent a component of the system that is not capable of individual existence (unlike land plant saltwort) and clearly react to all changes, including from the outside. The nutrition of aquatic plants occurs in the form of diffusion (percolation) and drift (mass transfer), which is accompanied by chemical reactions and translate the substance in a forward traveling wave with velocity higher than the rates of diffusion. Thus, the wave regime or fluctuations in the "water - plant" system and other contacting media begin to prevail. The importance of discovering the processes of self-diffusion and /or tunnel effects is enormous; they explain the spread of matter across borders, i.e. the ability to self-organization [5].

The chemical composition of PAH indicates a fundamental difference in the mechanisms of migration of hydrocarbons in plants compared to silt and soil formations. This is confirmed by the values of entropy and the nature of PAH groups obtained on the basis of cluster analysis. Two paragenetic groups consisting of light (2-3 rings) and heavy (4-5) PAHs are very clearly distinguished for the combined sample of PAH concentrations in waters, silts and soils. Most likely, the explanation for this effect is the prevailing role in the distribution of PAH physical and chemical properties of the compounds themselves (the most important are solubility, octanol number, and the Gibbs energy).

The mobility and migration from one medium to another is due to the synergistic effect of the medium itself - porosity, temperature and lipophilicity, etc. This is evidenced by the calculations of the correlation coefficients, which indicate a close relationship of physicochemical characteristics with PAH concentrations in silts. The most significant positive influence (as far as significance is concerned) is in the solubility coefficients, and in the values of hydrophobicity $\left(\mathrm{lg} \mathrm{K}_{\mathrm{ow}}\right)$ and the values of the Gibbs energy - negative bonds. The relationships between the enthalpy of formation of the gas phase and the solid substance, as well as the magnitudes of molecular entropy with variations in the concentration of PAHs, are markedly weaker. When going from point to point (from sample to sample), the degree of influence of physicochemical characteristics is different, which indicates the spatial diffusivity of the PAH composition.

These processes can change the tightness of PAH bonds, but the selected clusters will have a more or less stable structure, since the importance of their internal physicochemical properties that preserve the molecular structure prevails. Negative or close to zero values of entropy for waters, soils and silts on GCB confirm the above.

For higher plants, the obtained groups have a fundamentally different structure, indicating a change in the role of mechanisms of migration and transformation of PAHs. Here is unable to explain the process only the frequency of the external effects (e.g. 
pollution of PAHs through atmospheric deposition). Nevertheless, the endogenous mechanisms of pulsations, determined by the nature of interaction of the structural connections of the system and the possibility of self-organization outside the equilibrium (stationary state), are of great importance.

\section{Conclusion}

Estimates of the migration capacity of PAHs clearly indicate a variety of structural relationships between the components of the dissipative hydrogeochemical system formed by point sources - groundwater discharges. The diversity is caused by the initial chemical composition of water and the impulse nature of water release to the surface, which creates internal autowave processes in interaction with the "accepting" environment.

Considering the role of "living matter" in the GCS as a dissipative structure and active component significantly changes our understanding of nonequilibrium GCS at all levels of their organization.

Based on the experimental data, it seems important to supplement the classification of S.L. Shvartsev another one type of natural dissipative structures, developing with the participation of living matter.

The variety of substances passing from the solution directly into hydrophytes (as well as for terrestrial vegetation) is largely determined by the initial composition of the solution and the type of plants.

Calculations of entropy confirm the decisive role of living matter not only in the formation of surface formations during the hydrocarbon pollution genesis, but also in the causes of the formation of geochemical zonality during the migration of hydrocarbons from the surface to the aeration zone.

The maximum contribution to the formation of entropy (in absolute value) of the studied aquatic systems is made by GCB "soil - plant (root part)", followed by the border "plant (roots) - plant (stem)"; the minimum impact on the final value of entropy was made by the barrier "water - soil". This is due to a sharp change in the composition and concentration of PAH complex when moving them from soil to plant roots. Probably, PAHs get into a "friendly" environment (lipophilic compounds in the plant root cells) with minimal energy consumption.

The approach presented in the article allows to model and forecast behavior of organic matter in the aquatic system and to assess the accumulation of some pollutants in its components. Using these models, it is possible to understand the connections among the elements of aquatic systems and the direction of vary processes of migration of substances.

\section{References}

1. A.P. Khaustov, M.M. Redina, E.V. Yakovleva Geoekologiya. Inzhenernaya geologiya, gidrogeologiya, geokriologiya, 3, 3-17 (2018)

2. A.P. Khaustov, M.M. Redina, Geochemistry International, 55(1), 98-107 (2017)

3. S.L. Shvartsev Litosfera, 1, 65-89 (2007)

4. A.A. Oborin, S.A. Ilarionov, A.V. Nazarov et al. Oil-contaminated biogeocenoses (formation processes, scientific bases of restoration, medical and environmental problems), (Perm State University, Perm State Technical University, Ural Branch of RAS, Perm, 2008)

5. Khaustov, O. Korobova, M. Redina, International multidisciplinary scientific geoconference SGEM, 18, 869-874 (2018) 